\title{
Friend or Foe? The Varied Faces of Homeostatic Synaptic Plasticity in Neurodegenerative Disease
}

\author{
Henry B. C. Taylor and Alexander F. Jeans* \\ Department of Pharmacology, University of Oxford, Oxford, United Kingdom
}

Homeostatic synaptic plasticity (HSP) regulates synaptic strength both pre- and postsynaptically to ensure stability and efficient information transfer in neural networks. A number of neurological diseases have been associated with deficits in HSP, particularly diseases characterised by episodic network instability such as migraine and epilepsy. Recently, it has become apparent that HSP also plays a role in many neurodegenerative diseases. In this mini review, we present an overview of the evidence linking HSP to each of the major neurodegenerative diseases, finding that HSP changes in each disease appear to belong to one of three broad functional categories: (1) deficits in HSP at degenerating synapses that contribute to pathogenesis or progression; (2) HSP induced

OPEN ACCESS

Edited by:

Roberto De Pasquale,

University of São Paulo, Brazil

Reviewed by:

Marco Pignatelli,

Washington University in St. Louis,

United States

Matthew Philip Parsons,

Memorial University of

Newfoundland, Canada

${ }^{*}$ Correspondence:

Alexander F. Jeans

alexander.jeans@pharm.ox.ac.uk

Specialty section

This article was submitted to

Cellular Neurophysiology,

a section of the journal

Frontiers in Cellular Neuroscience

Received: 24 September 2021 Accepted: 19 November 2021 Published: 10 December 2021

Citation:

Taylor HBC and Jeans AF (2021) Friend or Foe? The Varied Faces of Homeostatic Synaptic Plasticity in Neurodegenerative

Disease.

Front. Cell. Neurosci. 15:782768. doi: 10.3389/fncel.2021.782768 in a heterosynaptic or cell non-autonomous manner to support the function of networks of which the degenerating synapses or cells are part; and (3) induction of HSP within the degenerating population of synapses to preserve function and to resist the impact of synapse loss. Understanding the varied manifestations of HSP in neurodegeneration will not only aid understanding mechanisms of disease but could also inspire much-needed novel approaches to therapy.

Keywords: neurodegeneration, synaptic plasticity, synaptic scaling, Alzheimer's, Parkinson's, Huntington's, amyotrophic lateral sclerosis

\section{INTRODUCTION}

The ability of physiological systems to respond to and resist perturbations is vital to their continued normal and healthy functioning. In addition to the well-understood homeostatic systems that allow for the maintenance of key variables such as temperature or blood pressure within set bounds, it has become apparent that there are a variety of mechanisms responsible for maintaining the function of neuronal circuits and networks within an appropriate range. The best-studied of these mechanisms are those of synaptic scaling, otherwise known as homeostatic synaptic plasticity (HSP), which adjust synaptic strengths across either the whole neuron or a functionally relevant subregion (e.g., dendritic branch) in order to prevent excessive strengthening or weakening of synapses by the potential positive feedback loop created by Hebbian plasticity (Davis, 2013; Vitureira and Goda, 2013). HSP is therefore a critical check that is required to maintain both neuronal health and the fidelity of information transfer (Turrigiano, 2012). HSP utilizes a number of mechanisms that partly overlap with those mediating other forms of synaptic plasticity; while most of these are intrinsic to neurons, under some conditions glial-secreted factors such as TNF $\alpha$ or IL-33 may also be required (Wang et al., 2021). 
In addition to HSP, a number of other homeostatic mechanisms can also be deployed to stabilise neuronal activity across individual cells and networks. These include regulation of synapse number (Kirov et al., 1999; Wierenga et al., 2006), regulation of intrinsic neuronal excitability (Marder and Goaillard, 2006; Turrigiano, 2011), metaplastic changes to the thresholds for induction of Hebbian plasticity in response to previous synaptic activity (Abraham, 2008) and shifting the balance of excitatory and inhibitory activity (E/I ratio) within networks (Maffei et al., 2004; Gonzalez-Islas and Wenner, 2006).

A number of neurological disease states have been associated with disruption of the mechanisms of HSP, including migraine (Welch, 2003), autism (Nelson and Valakh, 2015), epilepsy (Turrigiano, 2011) and Rett syndrome (Della Sala and Pizzorusso, 2014). HSP dysfunction has also been proposed as a significant factor in the development of schizophrenia (Dickman and Davis, 2009), and it appears to be particularly important in mood disorders occurring either alone or associated with other neurological conditions. Indeed, it is becoming clear that not only does modulation of HSP offer a potentially fruitful therapeutic approach to mood disorder treatment, but some of the most effective current treatments for these disorders actually work via this mechanism (Kavalali and Monteggia, 2020). Many of these conditions have in common a tendency towards episodic dysregulation of activity across neuronal networks, which might be expected if key homeostatic regulators of activity are compromised. For example, inherited mutations in the pore-forming subunit of the $\mathrm{Ca}^{2+}$ channel $\mathrm{Ca}_{\mathrm{V}} 2.1$, a key presynaptic effector of homeostatic synaptic plasticity (Jeans et al., 2017), can be an inherited cause of either migraine or epilepsy, which result from the aberrant, episodic depression or enhancement of activity, respectively, across networks (Haan et al., 2008).

It is increasingly becoming evident that neurodegenerative diseases constitute part of this group of conditions associated with deficits in HSP. Indeed, it has been recognised for some years that neuronal network-level function is pathologically unstable in several neurodegenerative diseases, potentially accounting for the day-to-day shifts in performance status that characterise many of them (Palop et al., 2006). This mini review will focus on the emerging literature supporting a role for HSP in the major neurodegenerative diseases and in particular highlight an intriguing dichotomy that is becoming apparent. In certain settings, as with non-degenerative neurological diseases, the failure of mechanisms of HSP likely contributes to pathogenesis and progression. Yet in others, HSP has a very different significance as it can be recruited to resist disease progression and confer a degree of protection against the loss of neuronal and network function.

\section{ALZHEIMER'S DISEASE}

Alzheimer's disease $(\mathrm{AD})$ is a substantial and increasing societal burden that is predicted to affect over 100 million people by 2050 (Prince et al., 2013). The disease is thought to be triggered by the pathological accumulation of soluble oligomers of amyloid $\beta$ (A $\beta$; Mucke and Selkoe, 2012), which exert a variety of effects on different cell types (De Strooper and Karran, 2016). Alterations in synaptic transmission are among the earliest observed effects, and these include enhanced excitatory activity in corticohippocampal networks (Busche and Konnerth, 2016) and impaired Hebbian synaptic plasticity at glutamatergic synapses, specifically a marked attenuation of long-term potentiation (LTP) and facilitation of long term depression (LTD; Walsh et al., 2002; Hsieh et al., 2006; Shankar et al., 2008; Li et al., 2009, 2011). Both of these phenomena precede the appearance of pathology and are considered to be the key cellular substrates of early cognitive impairment in AD.

There is a substantial body of evidence in support of the idea that deficiencies in HSP might contribute to the initiation and/or progression of $\mathrm{AD}$ pathogenesis. At the most fundamental level, functional dysregulation of a variety of key protein effectors of various aspects of HSP has been associated with the development of $\mathrm{AD}$. These proteins include $\beta$-secretase and presenilin 1 , which are responsible for different steps in the cleavage pathway that regulates the production of $A \beta$ from the amyloid precursor protein (APP). Changes in the activity of either due to $\mathrm{AD}$-causative mutations in the protein itself (presenilin 1), or mutations in the sequence within APP that is recognised and cleaved by the protein ( $\beta$-secretase), are associated with enhanced production of $\mathrm{A} \beta$ and consequently $\mathrm{AD}$ pathogenesis (Hutton and Hardy, 1997; Zhang et al., 2017). Presenilin 1 has also been shown to be required for Akt-dependent homeostatic upscaling of synaptic strength following chronic blockade of action potential firing with TTX (Pratt et al., 2011), while $\beta$-secretase is essential for homeostatic increases in synaptic strength in the visual cortex following dark exposure, as well as for normalization of synaptic transmission by subsequent exposure to light (Petrus and Lee, 2014). These observations might be at least partly explained by the role that $A \beta$ itself appears to play in HSP since a recent study has demonstrated that soluble, secreted $A \beta$, but not the parent protein APP nor other APP cleavage products, mediates the homeostatic upscaling of synaptic strength following TTX treatment in cultured neurons (Galanis et al., 2021). While this study suggests that $\mathrm{A} \beta$ may act in this context via postsynaptic mechanisms, other work indicates that it can regulate presynaptic function in response to chronically altered synaptic activity (Abramov et al., 2009), and the locus or loci of action of $A \beta$ in HSP, therefore, remains an open question.

Other HSP-associated proteins linked to AD include cyclindependent kinase 5 (CDK5), a pleiotropic effector acting at various intracellular locations that has been strongly implicated in $\mathrm{AD}$ pathogenesis. CDK5 becomes both inappropriately activated and delocalised following $\mathrm{A} \beta$ exposure (Patrick et al., 1999), phosphorylating a range of targets, including tau, that contribute to the synaptic decline and eventual neuronal loss (Shukla et al., 2012). CDK5 also homeostatically regulates synaptic strength following experimental neuronal silencing, acting via distinct presynaptic (Kim and Ryan, 2010) and postsynaptic (Seeburg et al., 2008) mechanisms. TNF $\alpha$ appears to play multiple roles in $\mathrm{AD}$ pathogenesis since it is present at elevated levels in $\mathrm{AD}$ brains, and inhibiting $\mathrm{TNF} \alpha$ signalling in a mouse model of $\mathrm{AD}$ lowered $\mathrm{A} \beta$ production and rescued 
cognitive deficits (Chang et al., 2017). TNF $\alpha$ of glial origin is also critical for the induction of HSP in response to chronic activity blockade (Stellwagen and Malenka, 2006). EphA4 is required for the synaptotoxic effects of oligomeric $A \beta$ (Vargas et al., 2014), and controls excitatory synaptic strength during HSP by regulating AMPAR levels via CDK5 activity (Fu et al., 2007, 2011; Peng et al., 2013). Conversely, repressor element-1 silencing transcription factor (REST) appears to play a neuroprotective role in AD. REST induction in the cortex and hippocampus is a part of normal ageing, and the protein acts as a potent protector from oxidative stress and $A \beta$-associated toxicity. However, REST is lost in $\mathrm{AD}$ brains, and conditional deletion of REST in mouse brains leads to age-related neurodegeneration (Lu et al., 2014). During HSP, REST reduces the strength of excitatory synapses in response to chronic network hyperactivity by acting presynaptically to decrease the size of functional synaptic vesicle pools (Pecoraro-Bisogni et al., 2018). These examples are all mediators of classical HSP; however, an even greater variety of proteins is implicated in both $\mathrm{AD}$ pathogenesis and more broadly defined processes of cellular and network homeostasis, and these have been well summarised elsewhere (Jang and Chung, 2016; Styr and Slutsky, 2018).

There is, therefore, a large body of evidence to support the idea that deficits in various components of the HSP machinery are essential for $\mathrm{AD}$ pathogenesis, although the evidence currently falls short of definitively placing HSP in a central role. However, a recently proposed hypothesis raises exactly this possibility by invoking HSP, together with non-synaptic homeostatic mechanisms, as a functional link between the two most consistently observed early neural signatures of $\mathrm{AD}$, enhanced excitatory activity in cortical and hippocampal networks and deficits in Hebbian synaptic plasticity (Styr and Slutsky, 2018).

A region-specific increase in tonic excitatory activity is one of the earliest abnormalities that can be observed in both $\mathrm{AD}$ patients (Mondadori et al., 2006; Filippini et al., 2009; Sperling et al., 2009; Bateman et al., 2012; Reiman et al., 2012) and animal models of the disease (Busche et al., 2008, 2012, 2015; Rudinskiy et al., 2012; Maier et al., 2014), usually preceding the emergence of overt pathology in $\mathrm{AD}$ patients by years (Busche and Konnerth, 2016). It appears to play a significant role in symptomatology and possibly pathogenesis, since reducing network hyperactivity pharmacologically has been shown to improve cognitive performance in patients with mild cognitive impairment (MCI), considered to be the prodromal stage of Alzheimer's (Bakker et al., 2012, 2015), and in AD model mice (Sanchez et al., 2012; Nygaard et al., 2015). In addition to simple hyperactivity, network hypersynchrony has been observed in $\mathrm{AD}$ animal models, resulting in epileptiform and seizure activity (Palop et al., 2007; Minkeviciene et al., 2009; Palop and Mucke, 2016). Consistent with this, AD patients are at elevated risk for epileptic seizures (Hauser et al., 1986; Romanelli et al., 1990), particularly those with autosomal dominant, early-onset familial AD (Palop and Mucke, 2016). The recently proposed "failure of firing homeostasis and plasticity" (FHP) hypothesis argues that such early changes are the result of a failure of homeostasis of network activity that arises when two necessary conditions have been met: firstly, some kind of perturbation of activity is imposed on susceptible brain networks, and secondly, that one or more of the core (meaning non-redundant) homeostatic mechanisms that would normally functionally compensate the perturbation are lost or compromised (Styr and Slutsky, 2018). As discussed above, the second of these criteria seems to be clearly met in $\mathrm{AD}$, since several mechanisms of HSP, including a number that normally serves to downscale synaptic strength, are dysregulated. The perturbation could be any of a variety of environmental or age-related phenomena that in healthy individuals are readily compensated; in $\mathrm{AD}$, drivers of the early network hyperactivity might include the build-up of $A \beta$, which is a positive regulator of neurotransmitter release (Abramov et al., 2009). According to the FHP hypothesis, the resultant chronically elevated networklevel activity might drive maladaptive, principally postsynaptic changes via various mechanisms, including mechanisms of HSP that remain intact. Synapse weakening and elimination in $\mathrm{AD}$ might, therefore, simply represent a functional compensation that is insufficient to renormalize hyperactivity (Styr and Slutsky, 2018). These events would almost certainly impact Hebbian plasticity, both because of its mechanistic overlap with HSP, and because of the likely recruitment of metaplasticity processes, which set thresholds for Hebbian plasticity in line with the recent history of activity at the synapse (Abraham, 2008). In summary, therefore, a primary failure in mechanisms regulating HSP could lead to chronically enhanced network activity in the initial stages of the disease, which in turn could drive compensatory synapse weakening and eventual elimination, together with the plasticity deficits (impaired LTP and facilitation of LTD) that are a key substrate of cognitive decline in the early stages of $\mathrm{AD}$ (Mucke and Selkoe, 2012; Styr and Slutsky, 2018; Figure 1). Accordingly, the overall scheme of $\mathrm{AD}$ pathogenesis appears to be one in which loss of HSP function leads to deleterious consequences for affected synapses.

While this account of AD pathogenesis is highly consistent with existing experimental data obtained in a variety of model systems and with clinical observations, there is an alternative possible interpretation of the current evidence. It remains possible that postsynaptic weakening is a primary event, possibly the result of pathological signalling downstream of postsynaptic binding of pathogenic conformations of $A \beta$ (Um et al., 2012; Kim et al., 2013), and this engages mechanisms of HSP to enhance presynaptic function and thereby synaptic activity in order to preserve synaptic strength (Taylor et al., 2020). Although this view seems much less favoured by the available evidence, it would again place HSP in an important role in pathogenesis, albeit as a maladaptive response to a pathological insult rather than as a primary, upstream event.

\section{PARKINSON'S DISEASE}

Like AD, Parkinson's disease (PD) is a progressive neurodegenerative disease that shows a strong association with age. The pathology of PD is principally characterised by the degeneration of the nigrostriatal tract composed of axonal projections from dopaminergic neurons located in the midbrain substantia nigra, and is a "dying-back" axonopathy in which 


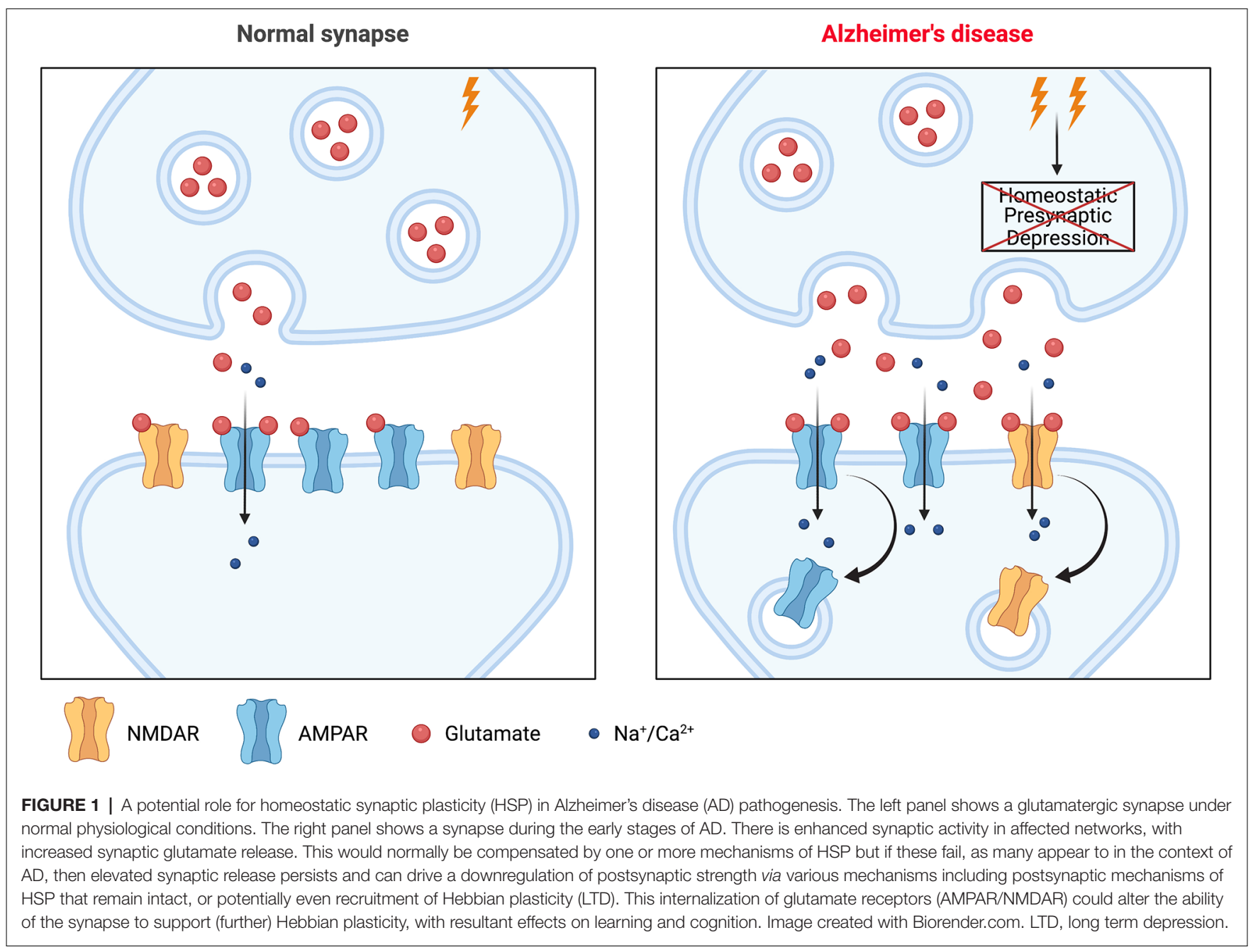

degeneration begins in the synaptic terminals and proceeds to the cell body (Burke and O'Malley, 2013). Clinically, PD presents with classical symptoms including bradykinesia, muscular rigidity and tremor; the first two of these are a direct result of dopaminergic neuron loss, while tremor is thought to relate to altered activity in neural circuits elsewhere in the brain (Helmich et al., 2012).

Somewhat analogous to $\mathrm{A} \beta$ in $\mathrm{AD}$, the key trigger for PD pathogenesis is thought to be an accumulation of the protein $\alpha$-synuclein (Stefanis, 2012). There is, however, a more limited body of evidence supporting the involvement of HSP in pathogenesis, at least in a cell-autonomous manner within the degenerating cell population. Physiologically, $\alpha$-synuclein regulates assembly of the presynaptic SNARE protein complex that controls neurotransmitter release (Chandra et al., 2005; Burre et al., 2010), and it is thought that in the context of pathology, $\alpha$-synuclein acts via both gain- and loss-of-function mechanisms to produce diverse effects including impairment of evoked neurotransmitter release in various PD model systems (Garcia-Reitbock et al., 2010; Nemani et al., 2010; Scott et al., 2010). The presynaptic localisation of $\alpha$-synuclein and its interaction with synaptic vesicles, together with the observations that it is trafficked within neurons in an activity-dependent manner (Fortin et al., 2005), and that its expression is regulated during learning in songbirds (George et al., 1995), all argue for a physiological role in synaptic plasticity. However, this appears not to be classical HSP, but more likely the maintenance of different functional pools of synaptic vesicles that are important for healthy synaptic function in the very long term (Burre, 2015). While there is, therefore, currently no clear evidence of an effect, either loss- or gain-of-function, on HSP in PD, this remains a possibility in view of the importance of $\alpha$-synuclein in synaptic regulation. Here, however, it is important to recognise that the mode of transmission at dopaminergic synapses is fundamentally different than that occurring at glutamatergic synapses of the sort affected in AD. The effects of dopamine at nigrostriatal synapses are mediated via volume transmission, in which the released neurotransmitter diffuses a relatively large distance to bind postsynaptic metabotropic receptors at multiple sites (Borroto-Escuela et al., 2018). This mode of synaptic transmission contrasts with the input specificity of glutamatergic synapses, which require independent regulation as well as co-regulation in functional groups of synapses across cells or networks. Indeed, it may be because of the need to balance 
these sometimes competing demands that HSP plays such an important role at glutamatergic synapses (Vitureira and Goda, 2013) and, conversely, may be less significant at dopaminergic synapses.

While there is little evidence for a role for HSP at the degenerating nigrostriatal synapses, there is evidence that dopamine depletion triggers the induction of a variety of heterosynaptic and cell non-autonomous homeostatic mechanisms within the striatum in order to preserve the function of basal ganglia circuits of which the degenerating synapses are part. These adaptations are principally seen in various classes of spiny projection neurons and include structural changes, such as changes in the density of dendritic spines (Stephens et al., 2005), changes in cellular excitability (Fieblinger et al., 2014) and changes in the ability of corticostriatal synapses to support Hebbian plasticity (Thiele et al., 2014). There is also evidence for changes in basal strength of striatal synapses following dopamine depletion, which are likely to represent typical HSP (Villalba and Smith, 2018). In particular, there is a specific reduction in the strength of glutamatergic corticostriatal synapses on to D2 receptor-expressing spiny neurons of the indirect pathway (Suarez et al., 2016), the inhibitory arm of the system that represents the major regulator of the initiation and amplitude of voluntary movements. This change appears to require the local upregulation of $\mathrm{TNF} \alpha$, an established mediator of HSP (Lewitus et al., 2014), and serves to maintain the strength of output from spiny neurons that would otherwise be pathologically enhanced by the loss of heterosynaptic D2R-mediated inhibition accompanying the loss of dopaminergic afferents (Figure 2A). In addition, there is a loss of both synaptic strength and connectivity amongst the recurrent collateral synapses that mediate inhibitory synaptic activity amongst the spiny neurons themselves (Taverna et al., 2008), although the functional significance of this is less clear.

These observations, therefore, suggest another potential role for HSP in neurodegenerative disease, quite distinct from its involvement in driving degeneration through loss of function as in $\mathrm{AD}$. In the context of $\mathrm{PD}$, HSP can be recruited in a heterosynaptic or cell non-autonomous manner at various loci of control within basal ganglia circuits to resist the effects of synaptic/neuronal loss, specifically the loss of dopaminergic nigrostriatal projections, elsewhere.

\section{HUNTINGTON'S DISEASE}

Huntington's disease (HD) is an inherited autosomal dominant neurodegenerative disorder caused by an elongation of the CAG repeat region of the gene encoding the huntingtin ( $\mathrm{Htt}$ ) protein (MacDonald et al., 1993). The disease usually manifests in middle age and is characterised initially by loss of motor coordination, frequently accompanied by evidence of cognitive impairment and psychiatric changes. These early symptoms develop into a hyperkinetic movement disorder and, usually, an overt dementia. The rate of decline and severity of symptoms is correlated with the length of the pathogenic CAG repeat. Like PD, HD is a condition that affects the basal ganglia, and the motor symptoms are a direct result of degeneration of a specific class of striatal spiny projection neurons, an effect that is thought to be mediated via a toxic gain-of-function of the mutant Htt (Ross and Tabrizi, 2011).

In common with other neurodegenerative diseases HD does, therefore, exhibit a degree of selective neuronal vulnerability (Fu et al., 2018). However, a variety of neuronal populations may be involved, which is perhaps expected since HD is a genetic disease, and all cells will express the toxic mutant Htt protein. Although the degeneration of striatal spiny neurons is perhaps the best-recognized feature of HD pathology, cortical neurons are also affected relatively early in the course of the disease, accounting for the high incidence of cognitive and psychiatric symptoms in HD (Ross and Tabrizi, 2011). Cortical neurons are much more accessible and tractable for study than striatal neurons and have yielded the strongest evidence to suggest dysregulation of HSP in HD. In one study, cultured cortical neurons from HD model mice were shown to lack the ability to induce HSP in response to network silencing, a phenotype which could be rescued by enhancing BDNF signalling (SmithDijak et al., 2019). In vivo, loss of HSP is a likely explanation for the failure of dendritic spines in the barrel cortex to adapt to the loss of sensory input as expected following whisker trimming (Murmu et al., 2015). There is also some evidence from human HD patients, with data from transcranial magnetic stimulation of the motor cortex revealing various abnormalities including impairments in LTP and LTD-like plasticity at cortical synapses that might partly reflect defective homeostatic scaling processes (Calabresi et al., 2016).

In addition, there is some indirect evidence for a role for HSP in Huntington's disease (HD), although this is less compelling. A study of the Htt interactome and of perturbation genes relevant for HD pathology endpoints showed enrichment of both datasets for HSP-related genes, suggesting that interactions of mutant Htt with HSP-related proteins may contribute to pathogenesis or progression in HD (Wang et al., 2017). Furthermore, the microRNA miR-485, expression of which is dysregulated in HD, plays a key regulatory role in HSP, again raising the possibility of some overlap between these processes (Cohen et al., 2011). Overall, therefore, the picture emerging is one of loss and/or dysregulation of function in pathways mediating HSP, although the pathogenic significance of this is not yet clear, as these effects remain relatively little explored. Given the importance of HSP in maintaining healthy neuronal and network function, it seems likely that these deficits will play a role in pathogenesis or progression of $\mathrm{HD}$, as well as possibly in the generation of motor symptoms, and HD would in this case belong to the broad functional class of HSP involvement as AD, where changes in HSP are deleterious. It does, however, remain possible, if unlikely, that the dysregulation of HSP is merely a bystander phenomenon that contributes little to pathogenesis, and further work will be required to resolve these issues.

\section{AMYOTROPHIC LATERAL SCLEROSIS}

Amyotrophic lateral sclerosis (ALS) is a progressive neurodegenerative disease principally affecting upper and lower motor neurons and presenting as progressive motor 
A
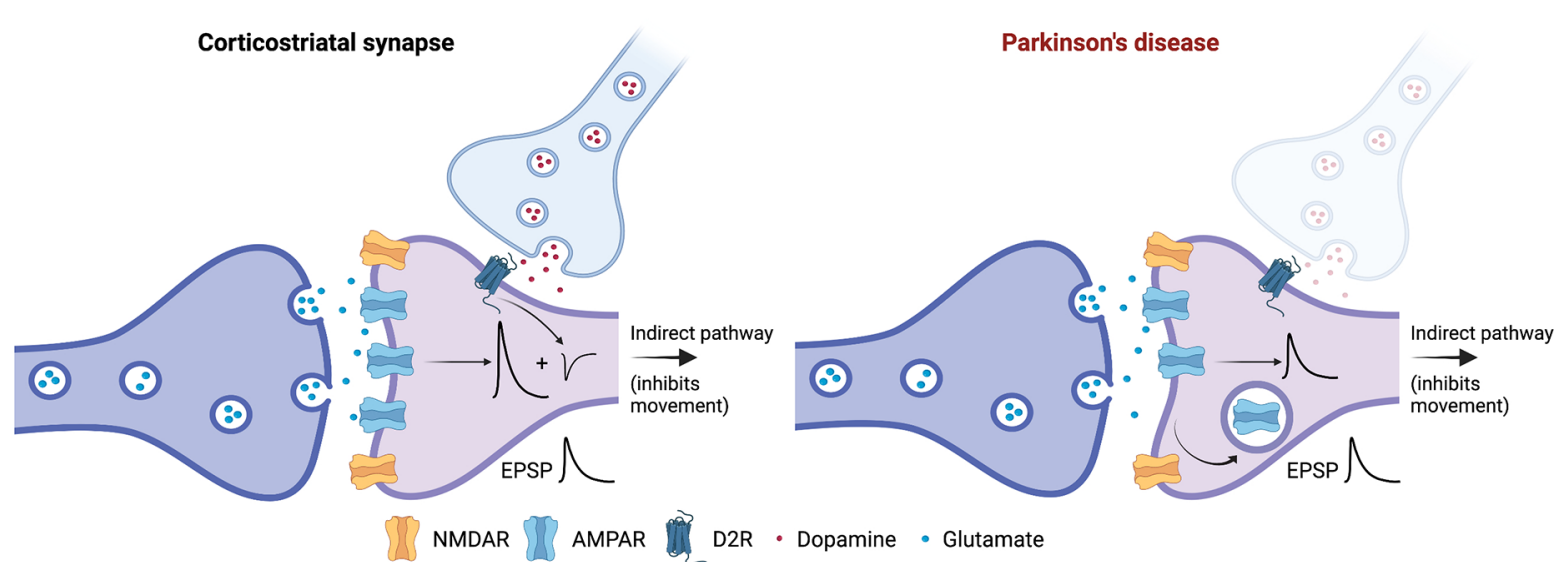

B

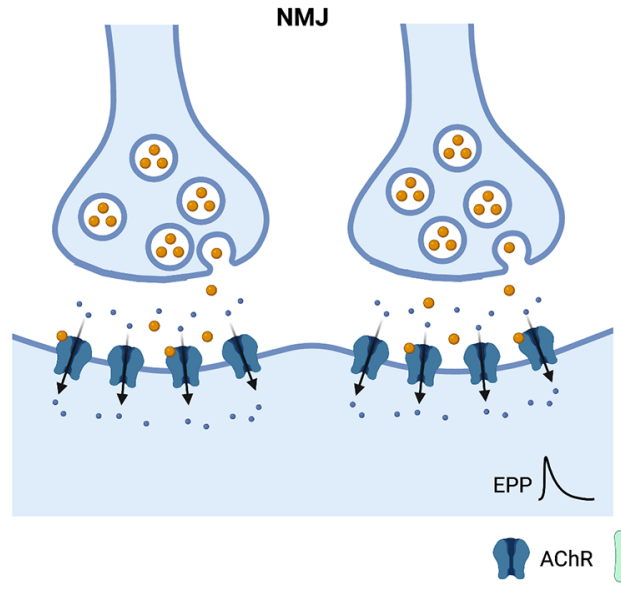

$L^{\mathrm{D} 2 \mathrm{R}}$

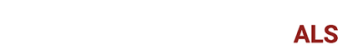

ALS

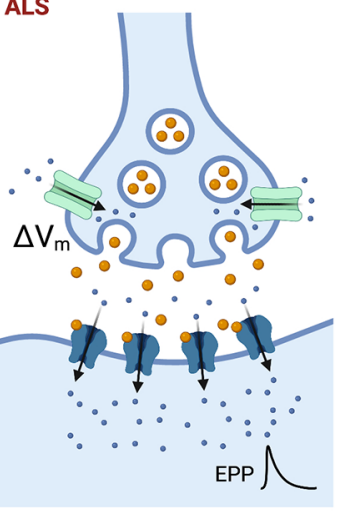

$\mathrm{ENaC}-\mathrm{ACh} \cdot \mathrm{Na}^{+}$

FIGURE 2 | Induction of homeostatic synaptic plasticity (HSP) can support the functioning of neuronal networks and/or populations of synapses in the context of synaptic degeneration. (A) Heterosynaptic and cell non-autonomous induction of HSP supports the function of basal ganglia circuits following degeneration of dopaminergic synapses in Parkinson's disease (PD). One example of this is shown involving synapses of the indirect pathway, which serves to negatively regulate voluntary movement. In models of PD, the strength of glutamatergic corticostriatal synapses onto medium spiny neurons is scaled down via mechanisms of HSP to compensate for the loss of D2 receptor-dependent inhibition (conceptually represented in the left panel as an inhibitory potential) of the same neuron by the degenerating nigrostriatal dopaminergic projections (right panel). Thus, heterosynaptic induction of HSP ensures that the overall strength of excitatory postsynaptic potentials (EPSPs) measured at the medium spiny neuron soma is preserved. (B) Induction of HSP at the degenerating neuromuscular junction (NMJ) supports motor function and opposes disease progression in amyotrophic lateral sclerosis (ALS) pathogenesis. The left panel shows the NMJ under physiological conditions. The right panel represents early stage ALS, in which innervation of the muscle and motor function is compromised by the loss of motor neuron synapses. This results in the induction of presynaptic HSP in remaining synaptic terminals via the membrane insertion of epithelial sodium channels (ENaC) that constitutively depolarise the presynaptic membrane $\left(\Delta \mathrm{V}_{\mathrm{m}}\right)$ and augment neurotransmitter release. This serves to: (1) preserve the strength of degenerating synapses; and/or (2) maintain normal or near-normal levels of postsynaptic depolarisation and end plate potentials (EPP) despite the loss of synaptic terminals, as shown here. Image created with Biorender.com.

deficits that develop over weeks or months. In later stages, there may also be cortical involvement with cognitive symptoms (van Es et al., 2017). Like other neurodegenerative diseases, the aetiology is not well understood but appears to be a complex mix of genetics and environmental factors. The greatest insights into pathogenesis thus far have come from the identification of a number of genes associated with familial variants of ALS, which group functionally into three main categories: RNA biology, protein turnover and axonal transport, suggesting that deficits in these processes play a causal role in pathogenesis (Renton et al., 2014). The discovery of causative genes has also assisted greatly with the production of suitable animal models of ALS for further mechanistic studies.
Because the most prominent feature is usually lower motor neuron degeneration with resultant muscular weakness, ALS is frequently thought of as a disease of the neuromuscular junction (NMJ; van Es et al., 2017). There is evidence in support of potential adaptive or homeostatic changes at NMJs in various animal models of ALS, and some in particular demonstrate enhanced spontaneous neurotransmission (Dzieciolowska et al., 2017; Bose et al., 2019), although it is not clear whether this is functionally significant or beneficial.

One intriguing feature of ALS, which is by no means unique to this disease, is that a significant loss of synapses can occur in affected regions, principally the NMJ, prior to the onset of symptoms (Moloney et al., 2014). This observation suggests that endogenous mechanisms may, to some degree, be 
recruited to preserve function in the face of reduced synaptic number, although until recently the existence and identity of such mechanisms had never been confirmed. In 2020, a study initiated at the Drosophila NMJ showed that classical presynaptic HSP is induced at degenerating NMJs and that it functionally opposes the effects of motor neuron degeneration and loss (Orr et al., 2020). Presynaptic HSP is known to depend on the presynaptically localised epithelial $\mathrm{Na}^{+}$channel, ENaC (Younger et al., 2013), which was the basis for most of the manipulations used in this study. The authors went on to demonstrate that presynaptic HSP is induced similarly in a mouse model of motor neuron degeneration (Orr et al., 2020), supporting its relevance in mammalian systems. These exciting results establish a new paradigm for HSP in neurodegenerative disease whereby it is induced within the degenerating population of synapses themselves to functionally oppose the effects of disease progression (Figure 2B). It remains unclear whether this process is initiated within individual terminals during the process of degeneration in order to augment their own declining function, or whether it may be initiated by orphan postsynaptic elements in regions of synapse loss to enhance release from remaining terminals nearby. Alternatively, both of these mechanisms may operate, and further work will be required to resolve the various possibilities. In addition to the functional rescue, Orr et al. demonstrated that the induction of HSP at NMJs actually arrests degeneration. They hypothesize that this might be because the loss of synaptic transmission could lead to a loss of trophic support (growth and survival factors) normally released by the innervated muscle (Orr et al., 2020).

\section{CONCLUSIONS}

The body of evidence in support of a role for HSP in a variety of neurodegenerative diseases is growing steadily. What, then, is the nature of this role? We have presented here an overview of the currently available evidence, which suggests that the significance of HSP may be distinctly different in each of the major diseases associated with neurodegeneration.

In this context, HSP appears able to function in three broad ways, notwithstanding that there may be more yet to be

\section{REFERENCES}

Abraham, W. C. (2008). Metaplasticity: tuning synapses and networks for plasticity. Nat. Rev. Neurosci. 9:387. doi: 10.1038/nrn2356

Abramov, E., Dolev, I., Fogel, H., Ciccotosto, G. D., Ruff, E., and Slutsky, I. (2009). Amyloid-beta as a positive endogenous regulator of release probability at hippocampal synapses. Nat. Neurosci. 12, 1567-1576. doi: 10.1038/nn.2433

Bakker, A., Albert, M. S., Krauss, G., Speck, C. L., and Gallagher, M. (2015). Response of the medial temporal lobe network in amnestic mild cognitive impairment to therapeutic intervention assessed by fMRI and memory task performance. Neuroimage Clin. 7, 688-698. doi: 10.1016/j.nicl.2015.02.009

Bakker, A., Krauss, G. L., Albert, M. S., Speck, C. L., Jones, L. R., Stark, C. E., et al. (2012). Reduction of hippocampal hyperactivity improves cognition in amnestic mild cognitive impairment. Neuron 74, 467-474. doi: 10.1016/j. neuron.2012.03.023

Bateman, R. J., Xionge, C., Benzinger, T. L. S., Fagan, A. M., Goate, A., Fox, N. C., et al. (2012). Clinical and biomarker changes in dominantly recognised. The first is a direct involvement in pathogenesis, usually when the failure of one or more mechanisms of HSP in degenerating synapses leads to consequences that drive the development of disease. A substantial body of evidence suggests that this is a critical mechanism underlying Alzheimer's disease, and it also seems likely to be relevant to Huntington's disease, although here there is currently much less evidence to support the conclusion. Secondly, HSP may be induced in a heterosynaptic or cell non-autonomous manner in connected neurons to preserve the function of networks of which the degenerating synapses are part, as may happen in Parkinson's disease. Thirdly and finally, HSP may be recruited within degenerating populations of synapses themselves to oppose functional decline and even mitigate synapse loss, as in amyotrophic lateral sclerosis.

Recognition of the diverse manifestations of HSP in the disease context will aid the development of new therapeutic approaches, which are acutely needed. These may aim to restore or replace a dysfunctional protein or pathway, or to further augment a beneficial homeostatic response. It may also be possible to initiate homeostatic responses in cells in which these may be dormant or not otherwise induced. Such an approach has recently been validated in a Drosophila model of C9orf72-associated motor neuron loss in which presynaptic HSP is normally inactive at degenerating NMJs, but can be experimentally induced to restore synaptic strength (Perry et al., 2017).

\section{AUTHOR CONTRIBUTIONS}

HT and AJ conceived the review and wrote and reviewed the manuscript. All authors contributed to the article and approved the submitted version.

\section{FUNDING}

AJ was supported in part by an Medical Research Council (MRC) Clinician Scientist Fellowship (G0802812).

inherited Alzheimer's disease. N. Engl. J. Med. 367, 795-804 doi: 10.1056/NEJMoa1202753

Borroto-Escuela, D. O., Perez De La Mora, M., Manger, P., Narvaez, M., Beggiato, S., Crespo-Ramirez, M., et al. (2018). Brain dopamine transmission in health and Parkinson's disease: modulation of synaptic transmission and plasticity through volume transmission and dopamine heteroreceptors. Front. Synaptic Neurosci. 10:20. doi: 10.3389/fnsyn.2018.00020

Bose, P., Armstrong, G. A. B., and Drapeau, P. (2019). Neuromuscular junction abnormalities in a zebrafish loss-of-function model of TDP-43. J. Neurophysiol. 121, 285-297. doi: 10.1152/jn.00265.2018

Burke, R. E., and O'Malley, K. (2013). Axon degeneration in Parkinson's disease. Exp. Neurol. 246, 72-83. doi: 10.1016/j.expneurol.2012.01.011

Burre, J. (2015). The synaptic function of alpha-synuclein. J. Parkinsons Dis. 5, 699-713. doi: 10.3233/JPD-150642

Burre, J., Sharma, M., Tsetsenis, T., Buchman, V., Etherton, M. R., and Sudhof, T. C. (2010). Alpha-synuclein promotes SNARE-complex assembly in vivo and in vitro. Science 329, 1663-1667. doi: 10.1126/science.1195227 
Busche, M. A., Chen, X., Henning, H. A., Reichwald, J., Staufenbiel, M., Sakmann, B., et al. (2012). Critical role of soluble amyloid-beta for early hippocampal hyperactivity in a mouse model of Alzheimer's disease. Proc. Natl. Acad. Sci. U S A 109, 8740-8745. doi: 10.1073/pnas.1206171109

Busche, M. A., Eichhoff, G., Adelsberger, H., Abramowski, D., Wiederhold, K. H., Haass, C., et al. (2008). Clusters of hyperactive neurons near amyloid plaques in a mouse model of Alzheimer's disease. Science 321, 1686-1689. doi: 10.1126/science.1162844

Busche, M. A., Grienberger, C., Keskin, A. D., Song, B., Neumann, U., Staufenbiel, M., et al. (2015). Decreased amyloid-beta and increased neuronal hyperactivity by immunotherapy in Alzheimer's models. Nat. Neurosci. 18, 1725-1727. doi: 10.1038/nn.4163

Busche, M. A., and Konnerth, A. (2016). Impairments of neural circuit function in Alzheimer's disease. Philos. Trans. R. Soc. Lond. B Biol. Sci. 371:20150429. doi: 10.1098/rstb.2015.0429

Calabresi, P., Pisani, A., Rothwell, J., Ghiglieri, V., Obeso, J. A., and Picconi, B. (2016). Hyperkinetic disorders and loss of synaptic downscaling. Nat. Neurosci. 19, 868-875. doi: 10.1038/nn.4306

Chandra, S., Gallardo, G., Fernandez-Chacon, R., Schluter, O. M., and Sudhof, T. C. (2005). Alpha-synuclein cooperates with CSPalpha in preventing neurodegeneration. Cell 123, 383-396. doi: 10.1016/j.cell.2005.09.028

Chang, R., Yee, K. L., and Sumbria, R. K. (2017). Tumor necrosis factor alpha Inhibition for Alzheimer's Disease. J. Cent. Nerv. Syst. Dis. 9:1179573517709278. doi: 10.1177/1179573517709278

Cohen, J. E., Lee, P. R., Chen, S., Li, W., and Fields, R. D. (2011). MicroRNA regulation of homeostatic synaptic plasticity. Proc. Natl. Acad. Sci. U S A 108, 11650-11655. doi: 10.1073/pnas.1017576108

Davis, G. W. (2013). Homeostatic signaling and the stabilization of neural function. Neuron 80, 718-728. doi: 10.1016/j.neuron.2013.09.044

De Strooper, B., and Karran, E. (2016). The cellular phase of Alzheimer's disease. Cell 164, 603-615. doi: 10.1016/j.cell.2015.12.056

Della Sala, G., and Pizzorusso, T. (2014). Synaptic plasticity and signaling in Rett syndrome. Dev. Neurobiol. 74, 178-196. doi: 10.1002/dneu.22114

Dickman, D. K., and Davis, G. W. (2009). The schizophrenia susceptibility gene dysbindin controls synaptic homeostasis. Science 326, 1127-1130. doi: 10.1126/science.1179685

Dzieciolowska, S., Drapeau, P., and Armstrong, G. A. B. (2017). Augmented quantal release of acetylcholine at the vertebrate neuromuscular junction following tdp-43 depletion. PLoS One 12:e0177005. doi: 10.1371/journal.pone. 0177005

Fieblinger, T., Graves, S. M., Sebel, L. E., Alcacer, C., Plotkin, J. L., Gertler, T. S., et al. (2014). Cell type-specific plasticity of striatal projection neurons in parkinsonism and L-DOPA-induced dyskinesia. Nat. Commun. 5:5316. doi: $10.1038 /$ ncomms6316

Filippini, N., MacIntosh, B. J., Hough, M. G., Goodwin, G. M., Frisoni, G. B., Smith, S. M., et al. (2009). Distinct patterns of brain activity in young carriers of the APOE-epsilon4 allele. Proc. Natl. Acad. Sci. U S A 106, 7209-7214. doi: 10.1073/pnas.0811879106

Fortin, D. L., Nemani, V. M., Voglmaier, S. M., Anthony, M. D., Ryan, T. A., and Edwards, R. H. (2005). Neural activity controls the synaptic accumulation of alpha-synuclein. J. Neurosci. 25, 10913-10921. doi: 10.1523/JNEUROSCI.292205.2005

Fu, W. Y., Chen, Y., Sahin, M., Zhao, X. S., Shi, L., Bikoff, J. B., et al. (2007). Cdk5 regulates EphA4-mediated dendritic spine retraction through an ephexin1-dependent mechanism. Nat. Neurosci. 10, 67-76. doi: $10.1038 / \mathrm{nn} 1811$

Fu, H., Hardy, J., and Duff, K. E. (2018). Selective vulnerability in neurodegenerative diseases. Nat. Neurosci. 21, 1350-1358. doi: 10.1038/s41593018-0221-2

Fu, A. K. Y., Hung, K. W., Fu, W. Y., Shen, C., Chen, Y., Xia, J., et al. (2011). APC(Cdh1) mediates EphA4-dependent downregulation of AMPA receptors in homeostatic plasticity. Nat. Neurosci. 14, 181-189. doi: 10. $1038 / \mathrm{nn} .2715$

Galanis, C., Fellenz, M., Becker, D., Bold, C., Lichtenthaler, S. F., Muller, U. C., et al. (2021). Amyloid-beta mediates homeostatic synaptic plasticity. J. Neurosci. 41, 5157-5172. doi: 10.1523/JNEUROSCI.1820-20.2021

Garcia-Reitbock, P., Anichtchik, O., Bellucci, A., Iovino, M., Ballini, C., Fineberg, E., et al. (2010). SNARE protein redistribution and synaptic failure in a transgenic mouse model of Parkinson's disease. Brain 133, 2032-2044. doi: 10.1093/brain/awq132

George, J. M., Jin, H., Woods, W. S., and Clayton, D. F. (1995). Characterization of a novel protein regulated during the critical period for song learning in the zebra finch. Neuron 15, 361-372. doi: 10.1016/0896-6273(95)90040-3

Gonzalez-Islas, C., and Wenner, P. (2006). Spontaneous network activity in the embryonic spinal cord regulates AMPAergic and GABAergic synaptic strength. Neuron 49, 563-575. doi: 10.1016/j.neuron.2006.01.017

Haan, J., Terwindt, G. M., van den Maagdenberg, A. M., Stam, A. H., and Ferrari, M. D. (2008). A review of the genetic relation between migraine and epilepsy. Cephalalgia 28, 105-113. doi: 10.1111/j.1468-2982.2007.01460.x

Hauser, W. A., Morris, M. L., Heston, L. L., and Anderson, V. E. (1986). Seizures and myoclonus in patients with Alzheimer's disease. Neurology 36, 1226-1230. doi: $10.1212 /$ wnl.36.9.1226

Helmich, R. C., Hallett, M., Deuschl, G., Toni, I., and Bloem, B. R. (2012). Cerebral causes and consequences of parkinsonian resting tremor: a tale of two circuits? Brain 135, 3206-3226. doi: 10.1093/brain/aws023

Hsieh, H., Boehm, J., Sato, C., Iwatsubo, T., Tomita, T., Sisodia, S., et al. (2006) AMPAR removal underlies Abeta-induced synaptic depression and dendritic spine loss. Neuron 52, 831-843. doi: 10.1016/j.neuron.2006.10.035

Hutton, M., and Hardy, J. (1997). The presenilins and Alzheimer's disease. Hum. Mol. Genet. 6, 1639-1646. doi: 10.1093/hmg/6.10.1639

Jang, S. S., and Chung, H. J. (2016). Emerging link between Alzheimer's disease and homeostatic synaptic plasticity. Neural Plast. 2016:7969272. doi: $10.1155 / 2016 / 7969272$

Jeans, A. F., van Heusden, F. C., Al-Mubarak, B., Padamsey, Z., and Emptage, N. J. (2017). Homeostatic presynaptic plasticity is specifically regulated by P/Q-type $\mathrm{Ca}(2+)$ channels at mammalian hippocampal synapses. Cell Rep. 21, 341-350. doi: 10.1016/j.celrep.2017.09.061

Kavalali, E. T., and Monteggia, L. M. (2020). Targeting homeostatic synaptic plasticity for treatment of mood disorders. Neuron 106, 715-726. doi: 10.1016/j. neuron.2020.05.015

Kim, S. H., and Ryan, T. A. (2010). CDK5 serves as a major control point in neurotransmitter release. Neuron 67, 797-809. doi: 10.1016/j.neuron.2010.08. 003

Kim, T., Vidal, G. S., Djurisic, M., William, C. M., Birnbaum, M. E., Garcia, K. C., et al. (2013). Human LilrB2 is a beta-amyloid receptor and its murine homolog PirB regulates synaptic plasticity in an Alzheimer's model. Science 341, 1399-1404. doi: 10.1126/science.1242077

Kirov, S. A., Sorra, K. E., and Harris, K. M. (1999). Slices have more synapses than perfusion-fixed hippocampus from both young and mature rats. J. Neurosci. 19, 2876-2886. doi: 10.1523/JNEUROSCI.19-08-02876.1999

Lewitus, G. M., Pribiag, H., Duseja, R., St-Hilaire, M., and Stellwagen, D. (2014). An adaptive role of TNFalpha in the regulation of striatal synapses. J. Neurosci. 34, 6146-6155. doi: 10.1523/JNEUROSCI.3481-13.2014

Li, S., Hong, S., Shepardson, N. E., Walsh, D. M., Shankar, G. M., and Selkoe, D. (2009). Soluble oligomers of amyloid Beta protein facilitate hippocampal long-term depression by disrupting neuronal glutamate uptake. Neuron 62 , 788-801. doi: 10.1016/j.neuron.2009.05.012

Li, S., Jin, M., Koeglsperger, T., Shepardson, N. E., Shankar, G. M., and Selkoe, D. J. (2011). Soluble Abeta oligomers inhibit long-term potentiation through a mechanism involving excessive activation of extrasynaptic NR2B-containing NMDA receptors. J. Neurosci. 31, 6627-6638. doi: 10.1523/JNEUROSCI.020311.2011

Lu, T., Aron, L., Zullo, J., Pan, Y., Kim, H., Chen, Y., et al. (2014). REST and stress resistance in ageing and Alzheimer's disease. Nature 507, 448-454. doi: $10.1038 /$ nature 13163

MacDonald, M. E., Ambrose, C. M., Duyao, M. P., Myers, R. H., Lin, C., Srinidhi, L., et al. (1993). A novel gene containing a trinucleotide repeat that is expanded and unstable on Huntington's disease chromosomes. Cell 72, 971-983. doi: 10.1016/0092-8674(93)90585-E

Maffei, A., Nelson, S. B., and Turrigiano, G. G. (2004). Selective reconfiguration of layer 4 visual cortical circuitry by visual deprivation. Nat. Neurosci. 7, 1353-1359. doi: 10.1038/nn1351

Maier, F. C., Wehrl, H. F., Schmid, A. M., Mannheim, J. G., Wiehr, S., Lerdkrai, C. et al. (2014). Longitudinal PET-MRI reveals beta-amyloid deposition and rCBF dynamics and connects vascular amyloidosis to quantitative loss of perfusion. Nat. Med. 20, 1485-1492. doi: 10.1038/nm.3734 
Marder, E., and Goaillard, J. M. (2006). Variability, compensation and homeostasis in neuron and network function. Nat. Rev. Neurosci. 7, 563-574. doi: 10.1038/nrn1949

Minkeviciene, R., Rheims, S., Dobszay, M. B., Zilberter, M., Hartikainen, J., Fulop, L., et al. (2009). Amyloid beta-induced neuronal hyperexcitability triggers progressive epilepsy. J. Neurosci. 29, 3453-3462. doi: 10.1523/JNEUROSCI.5215-08.2009

Moloney, E. B., de Winter, F., and Verhaagen, J. (2014). ALS as a distal axonopathy: molecular mechanisms affecting neuromuscular junction stability in the presymptomatic stages of the disease. Front. Neurosci. 8:252. doi: 10.3389/fnins. 2014.00252

Mondadori, C. R., Buchmann, A., Mustovic, H., Schmidt, C. F., Boesiger, P., Nitsch, R. M., et al. (2006). Enhanced brain activity may precede the diagnosis of Alzheimer's disease by 30 years. Brain 129, 2908-2922. doi: 10.1093/brain/awl266

Mucke, L., and Selkoe, D. J. (2012). Neurotoxicity of amyloid beta-protein: synaptic and network dysfunction. Cold Spring Harb. Perspect. Med. 2:a006338. doi: 10.1101/cshperspect.a006338

Murmu, R. P., Li, W., Szepesi, Z., and Li, J. Y. (2015). Altered sensory experience exacerbates stable dendritic spine and synapse loss in a mouse model of Huntington's disease. J. Neurosci. 35, 287-298. doi: 10.1523/JNEUROSCI.024414.2015

Nelson, S. B., and Valakh, V. (2015). Excitatory/inhibitory balance and circuit homeostasis in autism spectrum disorders. Neuron 87, 684-698. doi: 10.1016/j. neuron.2015.07.033

Nemani, V. M., Lu, W., Berge, V., Nakamura, K., Onoa, B., Lee, M. K., et al. (2010). Increased expression of alpha-synuclein reduces neurotransmitter release by inhibiting synaptic vesicle reclustering after endocytosis. Neuron 65, 66-79. doi: 10.1016/j.neuron.2009.12.023

Nygaard, H. B., Kaufman, A. C., Sekine-Konno, T., Huh, L. L., Going, H., Feldman, S. J., et al. (2015). Brivaracetam, but not ethosuximide, reverses memory impairments in an Alzheimer's disease mouse model. Alzheimers Res. Ther. 7:25. doi: 10.1186/s13195-015-0110-9

Orr, B. O., Hauswirth, A. G., Celona, B., Fetter, R. D., Zunino, G., Kvon, E. Z., et al. (2020). Presynaptic homeostasis opposes disease progression in mouse models of ALS-like degeneration: evidence for homeostatic neuroprotection. Neuron 107, 95-111.e6. doi: 10.1016/j.neuron.2020.04.009

Palop, J. J., Chin, J., and Mucke, L. (2006). A network dysfunction perspective on neurodegenerative diseases. Nature 443, 768-773. doi: 10 . 1038/nature05289

Palop, J. J., Chin, J., Roberson, E. D., Wang, J., Thwin, M. T., Bien-Ly, N., et al. (2007). Aberrant excitatory neuronal activity and compensatory remodeling of inhibitory hippocampal circuits in mouse models of Alzheimer's disease. Neuron 55, 697-711. doi: 10.1016/j.neuron.2007.07.025

Palop, J. J., and Mucke, L. (2016). Network abnormalities and interneuron dysfunction in Alzheimer disease. Nat. Rev. Neurosci. 17, 777-792. doi: 10.1038/nrn.2016.141

Patrick, G. N., Zukerberg, L., Nikolic, M., de la Monte, S., Dikkes, P., and Tsai, L. H. (1999). Conversion of p35 to p25 deregulates Cdk5 activity and promotes neurodegeneration. Nature 402, 615-622. doi: 10.1038/45159

Pecoraro-Bisogni, F., Lignani, G., Contestabile, A., Castroflorio, E., Pozzi, D., Rocchi, A., et al. (2018). REST-dependent presynaptic homeostasis induced by chronic neuronal hyperactivity. Mol. Neurobiol. 55, 4959-4972. doi: 10.1007/s12035-017-0698-9

Peng, Y. R., Hou, Z. H., and Yu, X. (2013). The kinase activity of EphA4 mediates homeostatic scaling-down of synaptic strength via activation of Cdk5. Neuropharmacology 65, 232-243. doi: 10.1016/j.neuropharm.2012.10.012

Perry, S., Han, Y., Das, A., and Dickman, D. (2017). Homeostatic plasticity can be induced and expressed to restore synaptic strength at neuromuscular junctions undergoing ALS-related degeneration. Hum. Mol. Genet. 26, 4153-4167. doi: $10.1093 / \mathrm{hmg} / \mathrm{ddx} 304$

Petrus, E., and Lee, H. K. (2014). BACE1 is necessary for experience-dependent homeostatic synaptic plasticity in visual cortex. Neural Plast. 2014:128631. doi: 10.1155/2014/128631

Pratt, K. G., Zimmerman, E. C., Cook, D. G., and Sullivan, J. M. (2011). Presenilin 1 regulates homeostatic synaptic scaling through Akt signaling. Nat. Neurosci. 14, 1112-1114. doi: 10.1038/nn.2893
Prince, M., Bryce, R., Albanese, E., Wimo, A., Ribeiro, W., and Ferri, C. P. (2013). The global prevalence of dementia: a systematic review and metaanalysis. Alzheimers Dement. 9, 63-75.e2. doi: 10.1016/j.jalz.2012.11.007

Reiman, E. M., Quiroz, Y. T., Fleisher, A. S., Chen, K., Velez-Pardo, C., JimenezDel-Rio, M., et al. (2012). Brain imaging and fluid biomarker analysis in young adults at genetic risk for autosomal dominant Alzheimer's disease in the presenilin 1 E280A kindred: a case-control study. Lancet Neurol. 11, 1048-1056. doi: 10.1016/S1474-4422(12)70228-4

Renton, A. E., Chio, A., and Traynor, B. J. (2014). State of play in amyotrophic lateral sclerosis genetics. Nat. Neurosci. 17, 17-23. doi: 10.1038/nn.3584

Romanelli, M. F., Morris, J. C., Ashkin, K., and Coben, L. A. (1990). Advanced Alzheimer's disease is a risk factor for late-onset seizures. Arch. Neurol. 47, 847-850. doi: 10.1001/archneur.1990.00530080029006

Ross, C. A., and Tabrizi, S. J. (2011). Huntington's disease: from molecular pathogenesis to clinical treatment. Lancet Neurol. 10, 83-98. doi: 10.1016/S1474-4422(10)70245-3

Rudinskiy, N., Hawkes, J. M., Betensky, R. A., Eguchi, M., Yamaguchi, S., SpiresJones, T. L., et al. (2012). Orchestrated experience-driven Arc responses are disrupted in a mouse model of Alzheimer's disease. Nat. Neurosci. 15, 1422-1429. doi: 10.1038/nn.3199

Sanchez, P. E., Zhu, L., Verret, L., Vossel, K. A., Orr, A. G., Cirrito, J. R., et al. (2012). Levetiracetam suppresses neuronal network dysfunction and reverses synaptic and cognitive deficits in an Alzheimer's disease model. Proc. Natl. Acad. Sci. U S A 109, E2895-E2903. doi: 10.1073/pnas.1121081109

Scott, D. A., Tabarean, I., Tang, Y., Cartier, A., Masliah, E., and Roy, S. (2010). A pathologic cascade leading to synaptic dysfunction in alpha-synuclein-induced neurodegeneration. J. Neurosci. 30, 8083-8095. doi: 10.1523/JNEUROSCI. 1091-10.2010

Seeburg, D. P., Feliu-Mojer, M., Gaiottino, J., Pak, D. T., and Sheng, M. (2008). Critical role of CDK5 and Polo-like kinase 2 in homeostatic synaptic plasticity during elevated activity. Neuron 58, 571-583. doi: 10.1016/j.neuron.2008.03. 021

Shankar, G. M., Li, S., Mehta, T. H., Garcia-Munoz, A., Shepardson, N. E., Smith, I., et al. (2008). Amyloid-beta protein dimers isolated directly from Alzheimer's brains impair synaptic plasticity and memory. Nat. Med. 14, 837-842. doi: 10.1038/nm1782

Shukla, V., Skuntz, S., and Pant, H. C. (2012). Deregulated Cdk5 activity is involved in inducing Alzheimer's disease. Arch. Med. Res. 43, 655-662. doi: 10.1016/j. arcmed.2012.10.015

Smith-Dijak, A. I., Nassrallah, W. B., Zhang, L. Y. J., Geva, M., Hayden, M. R., and Raymond, L. A. (2019). Impairment and restoration of homeostatic plasticity in cultured cortical neurons from a mouse model of huntington disease. Front. Cell. Neurosci. 13:209. doi: 10.3389/fncel.2019.00209

Sperling, R. A., Laviolette, P. S., O’Keefe, K., O’Brien, J., Rentz, D. M., Pihlajamaki, M., et al. (2009). Amyloid deposition is associated with impaired default network function in older persons without dementia. Neuron 63 , 178-188. doi: 10.1016/j.neuron.2009.07.003

Stefanis, L. (2012). alpha-synuclein in Parkinson's disease. Cold Spring Harb. Perspect. Med. 2:a009399. doi: 10.1101/cshperspect.a009399

Stellwagen, D., and Malenka, R. C. (2006). Synaptic scaling mediated by glial TNFalpha. Nature 440, 1054-1059. doi: 10.1038/nature04671

Stephens, B., Mueller, A. J., Shering, A. F., Hood, S. H., Taggart, P., Arbuthnott, G. W., et al. (2005). Evidence of a breakdown of corticostriatal connections in Parkinson's disease. Neuroscience 132, 741-754. doi: 10.1016/j. neuroscience.2005.01.007

Styr, B., and Slutsky, I. (2018). Imbalance between firing homeostasis and synaptic plasticity drives early-phase Alzheimer's disease. Nat. Neurosci. 21, 463-473. doi: 10.1038/s41593-018-0080-x

Suarez, L. M., Solis, O., Aguado, C., Lujan, R., and Moratalla, R. (2016). LDOPA oppositely regulates synaptic strength and spine morphology in D1 and D2 striatal projection neurons in dyskinesia. Cereb. Cortex 26, 4253-4264. doi: 10.1093/cercor/bhw263

Taverna, S., Ilijic, E., and Surmeier, D. J. (2008). Recurrent collateral connections of striatal medium spiny neurons are disrupted in models of Parkinson's disease. J. Neurosci. 28, 5504-5512. doi: 10.1523/JNEUROSCI.5493-07.2008

Taylor, H. B. C., Tong, R., Jeans, A. F., and Emptage, N. J. (2020). A novel optical quantal analysis of miniature events reveals enhanced frequency following 
amyloid beta exposure. Front. Cell. Neurosci. 14:564081. doi: 10.3389/fncel. 2020.564081

Thiele, S. L., Chen, B., Lo, C., Gertler, T. S., Warre, R., Surmeier, J. D., et al. (2014). Selective loss of bi-directional synaptic plasticity in the direct and indirect striatal output pathways accompanies generation of parkinsonism and 1-DOPA induced dyskinesia in mouse models. Neurobiol. Dis. 71, 334-344. doi: $10.1016 /$ j.nbd.2014.08.006

Turrigiano, G. (2011). Too many cooks? Intrinsic and synaptic homeostatic mechanisms in cortical circuit refinement. Annu. Rev. Neurosci. 34, 89-103. doi: 10.1146/annurev-neuro-060909-153238

Turrigiano, G. (2012). Homeostatic synaptic plasticity: local and global mechanisms for stabilizing neuronal function. Cold Spring Harb. Perspect. Biol. 4:a005736. doi: 10.1101/cshperspect.a005736

Um, J. W., Nygaard, H. B., Heiss, J. K., Kostylev, M. A., Stagi, M., Vortmeyer, A., et al. (2012). Alzheimer amyloid-beta oligomer bound to postsynaptic prion protein activates Fyn to impair neurons. Nat. Neurosci. 15, 1227-1235. doi: $10.1038 / \mathrm{nn} .3178$

van Es, M. A., Hardiman, O., Chio, A., Al-Chalabi, A., Pasterkamp, R. J., Veldink, J. H., et al. (2017). Amyotrophic lateral sclerosis. Lancet 390, 2084-2098. doi: 10.1016/S0140-6736(17)31287-4

Vargas, L. M., Leal, N., Estrada, L. D., Gonzalez, A., Serrano, F., Araya, K., et al. (2014). EphA4 activation of c-Abl mediates synaptic loss and LTP blockade caused by amyloid-beta oligomers. PLoS One 9:e92309. doi: 10.1371/journal. pone.0092309

Villalba, R. M., and Smith, Y. (2018). Loss and remodeling of striatal dendritic spines in Parkinson's disease: from homeostasis to maladaptive plasticity? J. Neural Transm. (Vienna) 125, 431-447. doi: 10.1007/s00702-017-1735-6

Vitureira, N., and Goda, Y. (2013). Cell biology in neuroscience: the interplay between Hebbian and homeostatic synaptic plasticity. J. Cell Biol. 203, 175-186. doi: $10.1083 /$ jcb. 201306030

Walsh, D. M., Klyubin, I., Fadeeva, J. V., Cullen, W. K., Anwyl, R., Wolfe, M. S., et al. (2002). Naturally secreted oligomers of amyloid $\beta$ protein potently inhibit hippocampal long-term potentiation in vivo. Nature 416, 535-539. doi: $10.1038 / 416535 a$

Wang, Y., Fu, A. K. Y., and Ip, N. Y. (2021). Instructive roles of astrocytes in hippocampal synaptic plasticity: neuronal activity-dependent regulatory mechanisms. FEBS J. [Online ahead of print]. doi: 10.1111/febs 15878

Wang, J. K. T., Langfelder, P., Horvath, S., and Palazzolo, M. J. (2017). Exosomes and homeostatic synaptic plasticity are linked to each other and to Huntington's, Parkinson's and other neurodegenerative diseases by databaseenabled analyses of comprehensively curated datasets. Front. Neurosci. 11:149. doi: 10.3389/fnins.2017.00149

Welch, K. M. A. (2003). Contemporary concepts of migraine pathogenesis. Neurology 61, S2-S8. doi: 10.1212/wnl.61.8_suppl_4.s2

Wierenga, C. J., Walsh, M. F., and Turrigiano, G. G. (2006). Temporal regulation of the expression locus of homeostatic plasticity. J. Neurophysiol. 96, 2127-2133. doi: 10.1152/jn.00107.2006

Younger, M. A., Muller, M., Tong, A., Pym, E. C., and Davis, G. W. (2013). A presynaptic ENaC channel drives homeostatic plasticity. Neuron 79, 1183-1196. doi: 10.1016/j.neuron.2013.06.048

Zhang, S., Wang, Z., Cai, F., Zhang, M., Wu, Y., Zhang, J., et al. (2017). BACE1 cleavage site selection critical for amyloidogenesis and Alzheimer's pathogenesis. J. Neurosci. 37, 6915-6925. doi: 10.1523/JNEUROSCI.0340-17. 2017

Conflict of Interest: The authors declare that the research was conducted in the absence of any commercial or financial relationships that could be construed as a potential conflict of interest.

Publisher's Note: All claims expressed in this article are solely those of the authors and do not necessarily represent those of their affiliated organizations, or those of the publisher, the editors and the reviewers. Any product that may be evaluated in this article, or claim that may be made by its manufacturer, is not guaranteed or endorsed by the publisher.

Copyright (c) 2021 Taylor and Jeans. This is an open-access article distributed under the terms of the Creative Commons Attribution License (CC BY). The use, distribution or reproduction in other forums is permitted, provided the original author(s) and the copyright owner(s) are credited and that the original publication in this journal is cited, in accordance with accepted academic practice. No use, distribution or reproduction is permitted which does not comply with these terms. 